IdeAs

Idées d'Amériques

13 | 2019

La photographie documentaire contemporaine dans les Amériques

\title{
Des friches et des ruines
}

Wastelands and ruins

Terrenos baldíos y ruinas

Danièle Méaux

\section{OpenEdition}

Journals

Édition électronique

URL : http://journals.openedition.org/ideas/5622

DOI : $10.4000 /$ ideas.5622

ISSN : $1950-570$

Éditeur

Institut des Amériques

Référence électronique

Danièle Méaux, «Des friches et des ruines », IdeAs [En ligne], 13 | 2019, mis en ligne le 01 mars 2019 consulté le 01 mai 2019. URL : http://journals.openedition.org/ideas/5622 ; DOI : 10.4000/ideas.5622

Ce document a été généré automatiquement le 1 mai 2019.

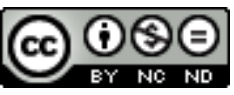

IdeAs - Idées d'Amériques est mis à disposition selon les termes de la licence Creative Commons Attribution - Pas d'Utilisation Commerciale - Pas de Modification 4.0 International. 


\title{
Des friches et des ruines
}

\author{
Wastelands and ruins \\ Terrenos baldíos y ruinas
}

Danièle Méaux

\section{Introduction}

1 Pour désigner le contexte socio-économique actuel, certains essayistes parlent de " période post-industrielle », tandis que d'autres recourent à l'expression de «troisième révolution industrielle » (Caron F., 1997). Si ces divergences terminologiques pointent des conceptions socio-économiques différentes, il reste que l'irruption des nouvelles technologies et la mondialisation des modes de production ont entraîné de profondes modifications sociales, une augmentation du chômage et le développement d'une économie de service. Par voie de conséquence, nombreux sont les équipements et les bâtiments, auparavant dédiés à l'industrie, qui sont aujourd'hui transformés en " grandes machines inutiles ", pour reprendre la formule de l'architecte italien Stefano Boeri (Boeri S., 2013 : 39). Un peu partout, des friches et des bâtiments abandonnés trouent le tissu urbain qui n'a plus la cohérence auparavant prêtée à la « ville » (Boeri S., 2013 : 39). Ce phénomène a d'importantes conséquences pour la vie des citadins, il pose problème aux responsables territoriaux et interroge géographes et urbanistes.

2 Ces équipements industriels désaffectés attirent les photographes, pour leur potentiel figural, mais aussi pour les questions économiques, sociales, politiques et urbanistiques qu'ils portent en creux. Bernd et Hilla Becher contribuèrent, par leurs typologies, à enclencher un intérêt du monde de l'art pour l'architecture industrielle; leurs travaux furent présentés dans la cadre de l'exposition New Topographics: Photographs of a Man Altered Lanscape en 1975, à Rochester. Mais leur attention se focalisait sur les formes d'un bâti dé-fonctionnalisé, non sur sa décrépitude. De nos jours, une attirance partagée pour le délabrement, la détérioration même des constructions modernes et industrielles s'est développée, au point de devenir quasiment une mode. Détroit se présente comme le hautlieu de ce «tourisme» un peu particulier. Les pratiques d'Urbex (Urban exploration) se 
multiplient, là et ailleurs, et les amateurs de friches et de ruines partagent leurs images sur les réseaux sociaux qui se présentent comme de nouveaux espaces d'exposition du spectaculaire.

Pourtant, de façon générale, les ruines de bâtiments modernes diffèrent des ruines romantiques qui renvoient à une époque glorieuse et reculée, car à la vision des pyramides, des églises gothiques ou des châteaux médiévaux à demi démolis, se trouve associée une méditation sur la fuite du temps et la finitude de la vie humaine, ainsi que purent l'exprimer Diderot ou encore Chateaubriand. En revanche, la visite d'usines délaissées et de hangars décatis ne donne pas à imaginer un temps long. Le passage de l'activité à la désuétude s'est joué en une période brève, puisque les cycles de renouvellement se font aujourd'hui sur un rythme de plus en plus rapide, comme l'a décrit le sociologue Harmut Rosa (2010). La décrépitude des ruines industrielles gagne ainsi en violence ce qu'elle perd en potentiel de suggestion métaphysique. Elle renvoie à des changements brutaux qui ont affecté l'existence d'individus dont certains sont aujourd'hui toujours en vie, et touche donc directement au contemporain.

Il va de soi que toute ruine possède une dimension indicielle forte (Caraion, M., 2003) : elle se présente comme le reste d'un usage passé auquel elle renvoie et la cause de la désuétude du bâti se trouve souvent suggérée par son état. L'agent de la détérioration a son importance : une ruine n'est pas perçue de la même manière selon qu'elle résulte de l'érosion exercée par des facteurs météorologiques sur une longue durée, de guerres qui mutilent les bâtiments comme on maltraite les corps ou encore d'une désaffection liée à des évolutions économiques. La perception d'un équipement ou d'un bâti désaffecté s'accompagne, dans la vie réelle, d'une conscience plus ou moins affirmée de sa vocation d'origine et des modalités de son processus de détérioration. Lorsque la ruine est mise en image, ce potentiel sémantique se trouve évidemment modulé par des paramètres formels divers. La photographie, qui est elle-même une empreinte, contribue en tout cas aisément à une exaltation de la dimension indicielle de la ruine, sa valeur de trace se trouvant pour ainsi dire portée au carré. Les clichés de bâtiments industriels désaffectés présentent donc une relation métonymique au chômage et à la misère, comme au déclin de tout un système économique (ils montrent les effets de son dysfonctionnement).

Toutefois, si pour toutes ces raisons la ruine industrielle diffère nettement de la ruine romantique, elle fournit néanmoins à l'opérateur des motifs, des agencements plastiques ou des variétés de texture qui ne sont pas éloignés de ceux qu'appréciait un William Gilpin à la fin du XVIII ${ }^{\mathrm{e}}$ siècle : le bâti se trouve fréquemment rattrapé par la végétation, les lignes et les formes peuvent être interrompues, trouées par l'érosion, par le pillage ou la destruction (Gilpin W., 1982 [1799]). L'usure tend à rendre les matières rugueuses, râpeuses et irrégulières, tandis que des dissymétries accidentelles apparaissent. Ces traits «objectifs» - confinant au pittoresque - peuvent être diversement traités par la photographie et la charge sémantique des clichés s'en trouve infléchie.

\section{Le spectacle de la catastrophe}

6 La ville de Détroit connait, entre 1900 et 1930, un formidable essor économique. Elle devient alors capitale mondiale de l'automobile: vers ses usines affluent les matières premières, tandis que des produits finis en partent pour être vendus dans le monde entier. La cité attire massivement les travailleurs migrants, de sorte que la croissance 
démographique est spectaculaire : en 1950, Détroit atteint 2 millions d'habitants. Les constructions ambitieuses se multiplient. L'architecte Albert Kahn travaille aussi bien pour les entreprises ou les institutions que pour les particuliers. Berceau du modèle fordiste, la ville est surnommée Motor Town ou Motor City. Son urbanisme, particulièrement étalé, tient à l'usage intensif de l'automobile - selon une logique qui tend d'ailleurs à organiser l'ensemble du territoire américain.

7 À partir des années 1950, la réduction de la main d'œuvre, la concurrence des constructeurs allemands ou japonais et les délocalisations engendrent une récession progressive de l'activité. L'augmentation du chômage et le déclin démographique sont vertigineux, dans la seconde moitié du $\mathrm{xx}^{\mathrm{e}}$ siècle. Le centre-ville - Downtown - se vide de ses habitants blancs et aisés qui partent vivre en périphérie, tandis qu'au cœur de la cité demeurent majoritairement les Afro-Américains. Les tensions croissent entre les communautés et des émeutes raciales d'une extrême violence éclatent en 1967, cette situation accentuant en cercle vicieux l'exode des blancs vers l'extérieur. Au centre-ville, les commerces ferment, les pertes de recettes entraînent l'effondrement des organismes de service public, la criminalité croît. En 2013, Détroit est une des premières villes américaines à être déclarée en faillite, avec une dette de 18 milliards de dollars. Aujourd'hui, le cœur de la cité est émaillé de ruines et de bâtiments abandonnés. La démolition de certains édifices laisse place à des terrains vagues.

8 La situation sociale et urbanistique est très préoccupante, mais la ville n'est pas sans "photogénie », au sens où elle peut produire, au cinéma comme en photographie, « un effet supérieur à l'effet produit au naturel », selon la définition du Petit Robert. De fait, Détroit attire aujourd'hui massivement les gens d'image et, afin de caractériser le caractère compulsif et spectaculaire de leurs prises de vue, certains commentateurs ont parlé de «Ruin Porn ». Selon des styles très différents, le chilien Camilo José Vergara ou le français Thomas Jorion a multiplié les images des ruines - bon nombre d'entre elles étant réalisées à Détroit (Vergara C., 2003 et son site ; Jorion Th., 2010).

Les travaux des français Yves Marchand et Romain Meffre ont particulièrement retenu l'attention. C'est en duo que travaillent ces photographes, avec une chambre $4 \times 5$ inches, décidant ensemble des choix techniques et esthétiques. Le livre qu'ils publient en 2010, résulte de cinq années de travail mené en commun à Détroit (Marchand Y., 2010). Leurs négatifs sont peu contrastés de sorte qu'ils retiennent une grande richesse de détails; les textures grenues accrochent la lumière ; la matérialité des objets érodés et les variations tonales sont rendues avec précision afin que rien ne soit perdu de l'état des bâtiments, de l'usure des matériaux et des surfaces; la profondeur de champ est importante; un maximum d'informations se trouve livré sur le délabrement affectant la moindre parcelle des espaces mis en image. C'est l'emphase et la saturation de l'ensemble qui frappent d'abord le lecteur/spectateur. À l'exception de quelques-unes d'entre elles, les très nombreuses images réunies sont présentées en pleine page et en assez grande taille

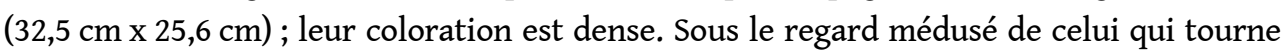
les pages de l'ouvrage, se succèdent les façades à demi démolies, les intérieurs délabrés, les friches envahies par les herbes et les murs décrépis. La quantité clame en elle-même l'ampleur d'un désastre atterrant.

10 À l'instar de certaines photographies réalisées après les accidents de Tchernobyl ou de Fukushima, ces vues de Détroit prennent une allure post-apocalyptique : dans les salles de classe, les bibliothèques ou les ateliers d'usine, l'activité semble s'être arrêtée brutalement; des objets épars se présentent comme les indices pétrifiés d'une vie 
soudainement interrompue. Dans un cabinet de dentiste, le matériel technique est toujours en place au milieu des gravats, comme si la roulette venait de quitter la bouche d'un patient (Marchand Y., 2010: 57). Quelques livres cornés en voie de décomposition gisent sur le sol d'une bibliothèque qui semble encore prête à accueillir des usagers (ibid. : 167). En une violente syncope, «l'Autrefois rencontre le Maintenant», pour reprendre une expression de Walter Benjamin (Benjamin W., 1989 :478). Les aiguilles arrêtées d'une horloge dont le cadran est à demi fondu pointent un horaire, jusqu'à nos jours suspendu (Marchand Y., 2010 : 101).

11 Enfin, le faste d'un certain nombre d'édifices (salles de concert, hôtels luxueux, immeubles de standing, etc.) comme le gigantisme des bâtiments industriels (usines, entrepôts, gares, etc.) tranche avec le degré avancé de leur délabrement, et cet écart renforce l'intensité du sentiment de la catastrophe. Certaines vues, figurant de somptueux théâtres complètement dévastés, semblent se rapprocher de l'oxymore (ibid. :77). En tout cas, la saturation en informations, le nombre des vues et des ruines, la pétrification de l'activité, le rapprochement brutal de la magnificence perdue et de la décrépitude actuelle se présentent comme les éléments d'une rhétorique dont l'efficacité est incontestable. Le lecteur/spectateur est sidéré par l'ampleur et la fulgurance du déclin. En raison de son étendue, la récession prend les allures d'un cataclysme ou d'un raz-de-marée: sa puissance confine à celle d'un désastre naturel - ce qui menace d'occulter sa dimension politique.

12 Les photographies réunies dans Détroit, vestige du rêve américain renvoient au naufrage de l'American Dream, ainsi que l'explicite le titre. Elles montrent le résultat d'une tragédie qui signe la faillite d'un modèle de progrès économique, et plus largement peut-être le deuil des « grands récits» (Lyotard J. F., 1979). Sans doute la fascination un peu morbide que suscitent ces images rencontre-t-elle l'attraction que les médias cultivent aujourd'hui pour l'imminence de la catastrophe et la conviction d'une vulnérabilité accrue de la planète (Lussault M., 2013:193-221; Virilio P., 2002). En ce sens, les images d'Yves Marchand et Romain Meffre, sacrifiant à certains penchants de masse et ne lésinant pas sur les moyens mis en œuvre pour les satisfaire, comportent une certaine dose de kitsch (Adorno Th., 1974 : 133).

13 Les murs sont craquelés, les plafonds éventrés, les sols jonchés de détritus; les façades s'écroulent, l'humidité ronge les matériaux ; les tonalités sont celles de la rouille et de la pourriture. La ruine, qui captive pour ses formes et ses coloris, revêt le caractère envoûtant d'un grand opéra et l'intérêt du spectateur s'absorbe dans l'effet rétinien des détériorations accumulées. En proportion de l'affirmation de la puissance iconique du phénomène, l'attention portée à son "indicialité » s'affaiblit: le spectacle obnubile l'observateur qui cherche moins la cause d'un processus qu'il ne tend à s'abîmer dans la contemplation d'un résultat. Alors que les ruines ont tendance à présenter une incomplétude qui est à même de déclencher l'imagination, engendrant le désir de remonter vers le passé, amorçant une sorte de pulsion de reconstitution, les formes du bâti décati s'imposent ici dans leur suffisance intrinsèque. Elles saturent l'attention et sidèrent le spectateur.

\section{L'expérience d'un paysage en devenir}

14 Les photographies réalisées par Joel Sternfeld sur le parcours de la High Line à New York - qui ont également pour objet une friche industrielle - obéissent à une esthétique bien 
différente. Elles renvoient à un arpentage du site, propice à une rêverie susceptible de faire ressurgir un usage antérieur et d'engendrer le projet d'un éventuel avenir.

$\mathrm{Au}$ tout début $\mathrm{du} \mathrm{xx}^{\mathrm{e}}$ siècle, le trafic est complètement saturé, sur la rive très industrialisée de l'Hudson, du côté Ouest de Manhattan, de sorte que la construction d'une voie aérienne est envisagée. À partir de 1934, les trains traversent les pâtés d'immeubles pour acheminer les marchandises vers les entrepôts et les usines qui sont alors prospères. Mais déjà, dans les années 1950-1960, le fret ferroviaire diminue au profit du transport routier et l'industrie tend à décliner. En 1980, circule le dernier train et la High Line devient une friche envahie par les herbes folles, abandonnée aux chats errants, aux oiseaux et aux insectes qui y pullulent. À ce moment-là, certains souhaitent purement et simplement sa démolition.

16 Mais des habitants du quartier se regroupent pour tenter de sauver la structure et d'en faire un équipement public. Ils souhaitent son réaménagement en un parc urbain suspendu, sur le modèle de la « Promenade plantée » réalisée à Paris en 1988 sur le tracé de l'ancienne voie ferroviaire de Vincennes. Se succèdent alors les étapes d'un patient travail de lobbying émaillé d'actions judiciaires et d'opérations de communication (Joshua D., 2011). C'est dans le cadre de cette entreprise collective que Joel Sternfeld, déjà reconnu pour ses travaux en couleur consacrés au paysage, est sollicité pour réaliser un ensemble de photographies de la friche. Il pratique ses prises de vue à la chambre, entre avril 2000 et juillet 2001, consignant ainsi les changements de saisons. Des photographies sont publiées dans le New Yorker, tandis que d'autres sont exposées; un ouvrage intitulé Walking the High Line parait chez Steidl (Sternfeld J., 2001). Le travail de Joel Sternfeld a incontestablement joué un rôle pour la défense du site et, à cet égard, il peut être comparé à celui qui fut conduit par William Henry Jackson en 1870 dans le Yellowstone et qui contribua à la transformation du lieu en parc national.

17 En 2004, la ville (dont le maire est désormais Michael Bloomberg) donne son accord pour le financement du réaménagement de la voie surélevée en promenade. C'est le projet soumis par James Corner Field Operations et Diller Scofidio + Renfro qui est retenu. Les travaux commencent en 2006 pour s'achever en 2009. La promenade, qui est alors ouverte au public, diffère d'autres parcs urbains à cause de la manière dont elle fend la ville en hauteur, mais aussi parce que le paysage nouvellement créé conserve pour partie le charme de la friche - telle qu'elle a été précisément photographiée par Joel Sternfeld. Elle garde en tout cas l'étagement des différents états connus par le site. Si sa fonction est récréative, l'aménagement réalisé favorise aussi la rétention des eaux de pluie ; il fournit de l'ombre, de l'oxygène et un lieu d'habitation pour les oiseaux et les insectes; il amène ainsi une forme de régénérescence naturelle à la ville de New York.

Après la désaffection de la voie, la végétation avait repris le pouvoir, ainsi que le montrent les vues de Joel Sternfeld: les herbes folles, les plantes vivaces envahissaient voies et ballast. Le point de vue adopté par le photographe - celui d'un homme qui marche - fait ressortir la taille et la vigueur des plantes, la variété des espèces végétales. Les images montrent même parfois des arbustes qui poussent entre les rails. Joel Sternfeld revient sur les lieux en été, en automne, en hiver, puis au printemps et ce parti pris met en valeur la vie végétale et les cycles naturels. Au sein de la flore, la faune n'est certainement pas absente, même si les images ne la montrent pas. Les variations saisonnières sont d'ordinaire plus perceptibles à la campagne qu'en ville où elles se trouvent concurrencées, masquées pour ainsi dire par d'autres rythmes (Paquot Th., 2011 : 117-132). La mise en évidence de l'alternance des saisons, au travers des variations 
météorologiques et des transformations végétales, engendre le sentiment d'un retour en force de la nature.

Figure 1 : Joel Sternfeld, $A$ Peach Tree, October 2000.

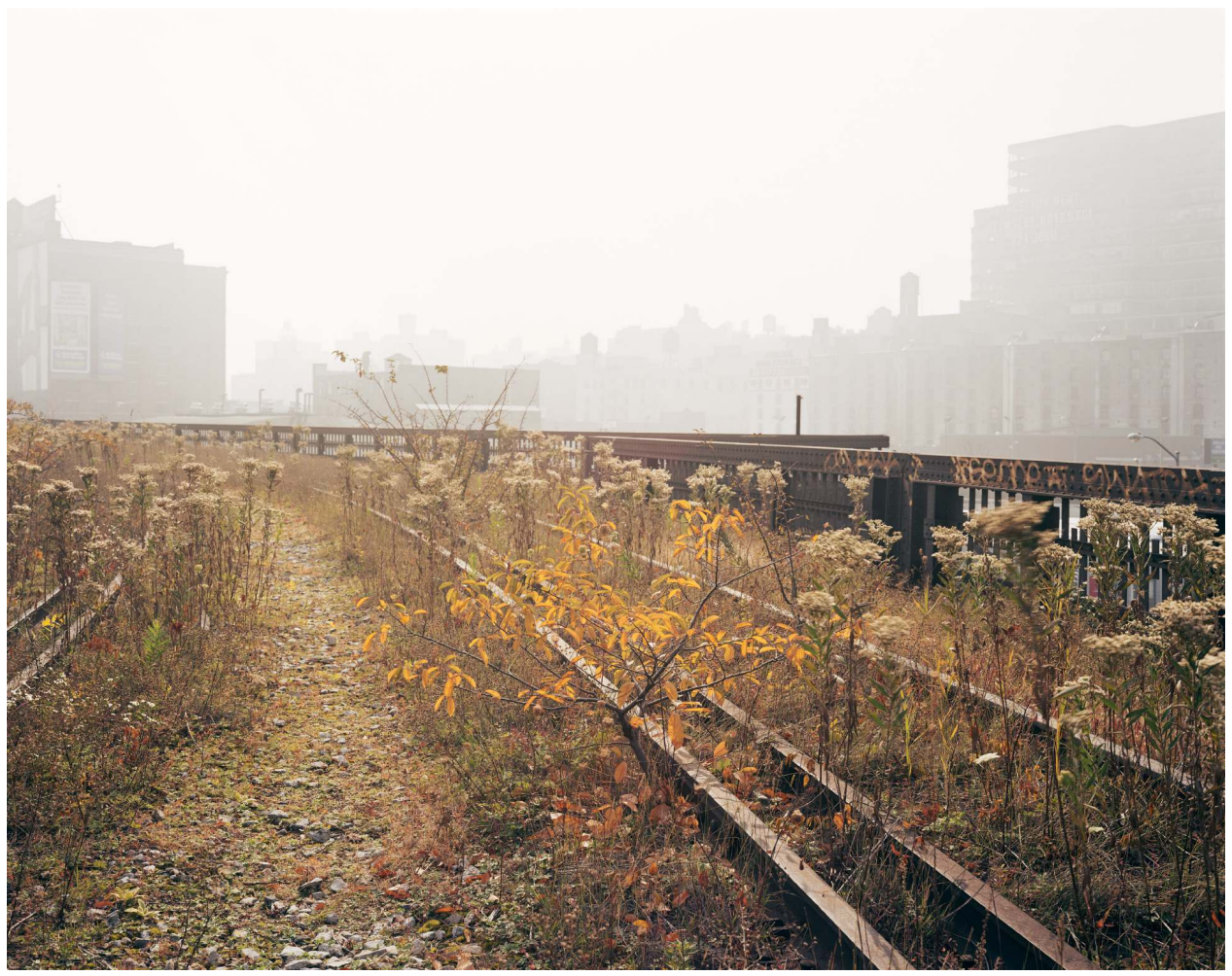

(c) Joel Sternfeld; Courtesy of the artist and Luhring Augustine, New York.

Dans les images de Joel Sternfeld, le traitement des couleurs est subtil : les tons dorés de l'herbe à la fin de l'été, les nuances chromatiques variées des fleurs fragiles au printemps voisinent le coloris des rails rouillés ; la lumière, douce et fluide, circule entre les tiges entremêlées, presque translucides parfois, devant les façades de briques usées. La végétation vient interrompre les formes régulières du bâti. Ces vues, de format " paysage ", confinent au pittoresque, en cultivant l'incomplet, le rugueux, l'accidenté. Cependant, à la différence des estampes des XVIII et XIX ${ }^{e}$ siècles, les photographies, qui sont absolument vides de présence humaine, refusent l'anecdote. Elles rappellent le caractère transitoire de la matière, le temps qui passe, renversant les prospérités passagères et les utopies de progrès économique. La ruine des constructions passées - que la poussée de la nature peu à peu submerge - renvoie à la notion «d'entropie » dont Robert Smithson a fait un de ses concepts essentiels (Smithson R., 1979). La désaffection, qui permet à la nature de reprendre le dessus, s'apparente à une catharsis puisqu'elle semble rompre avec le passé pour permettre un nouveau départ. La ruine et la friche se font ainsi allégorie d'un paysage en devenir. 
Figure 2 : Joel Sternfeld, A View towards the Hudson, Late February, 2001.

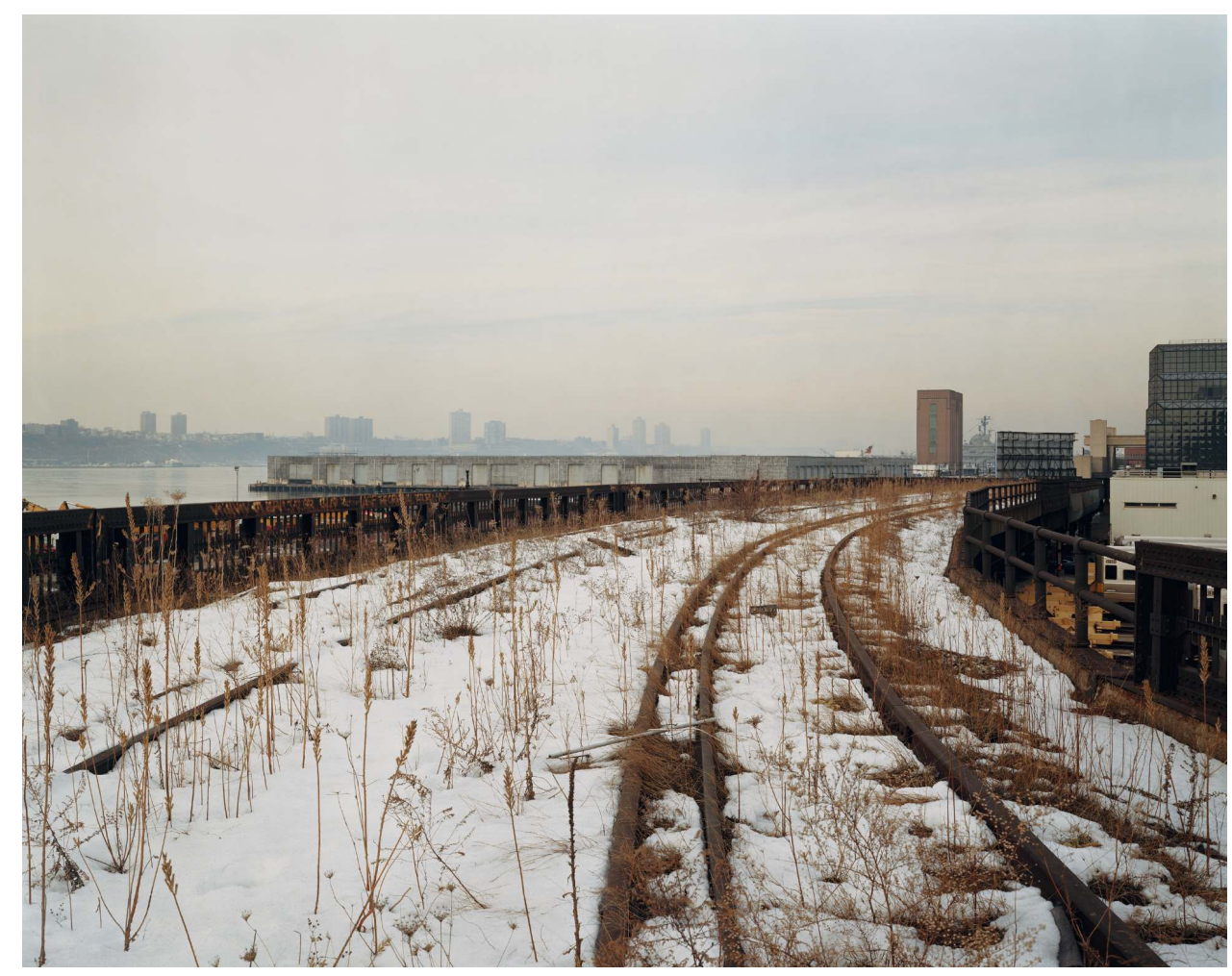

(c) Joel Sternfeld; Courtesy of the artist and Luhring Augustine, New York.

Alors que le peintre ou l'écrivain peuvent composer dans leur atelier ou à leur table de travail, le photographe est bel et bien obligé de se rendre sur le motif. L'exercice de la prise de vue s'allie la plupart du temps à une pratique du déplacement et, à bien des égards, Joel Sternfeld le met en évidence (Méaux D., 2009). Sa déambulation sur la High Line s'avère contrainte par le tracé de la voie : la suite de plans d'ensemble, réunis dans le livre, renvoie à une progression pédestre dans l'axe de la chaussée davantage qu'à une divagation erratique; l'expérience du photographe parait ainsi plus proche de celle de l'arpenteur que de celle du flâneur et son corps se fait instrument de mesure de l'espace.

21 À l'exception de la première vue, les images proposées dans Walking the High Line sont réalisées face au tracé de la voie. Les rails s'ouvrent devant le photographe (et donc le lecteur/spectateur), circonscrivant ainsi un cône qui enserre son regard et se fait invitation à une avancée dans l'espace. La profondeur de champ étant conséquente, les vues sont nettes des cailloux ou des rails qui se présentent au premier plan, près de l'endroit où le photographe a dû se tenir, jusqu'à l'horizon vers lequel il se dirige. C'est la position de l'opérateur qui détermine l'organisation du champ et sa progression renouvelle constamment l'aspect de la voie surélevée parcourue. L'épaisseur d'espace représenté dans chaque image renvoie à la durée nécessaire à la marche (Merleau Ponty H., 1985 : 306), et la succession des images, dans le dispositif livresque, suggère cette progression.

L'état de la friche fait qu'il est impossible d'y utiliser un véhicule. L'avancée est donc nécessairement pédestre et la marche - dont la suite des vues suggère le rythme - permet une immersion, une imprégnation minutieusement consentie. Elle suppose une durée 
incarnée dans des points de vue successifs correspondant à des positions physiques occupées par l'opérateur les unes après les autres. Cette dernière croise le temps cyclique des saisons qui transforment l'apparence du site.

Figure 3 : Joel Sternfeld, Ailanthus Trees, $25^{\text {th }}$ Street, May 2000.

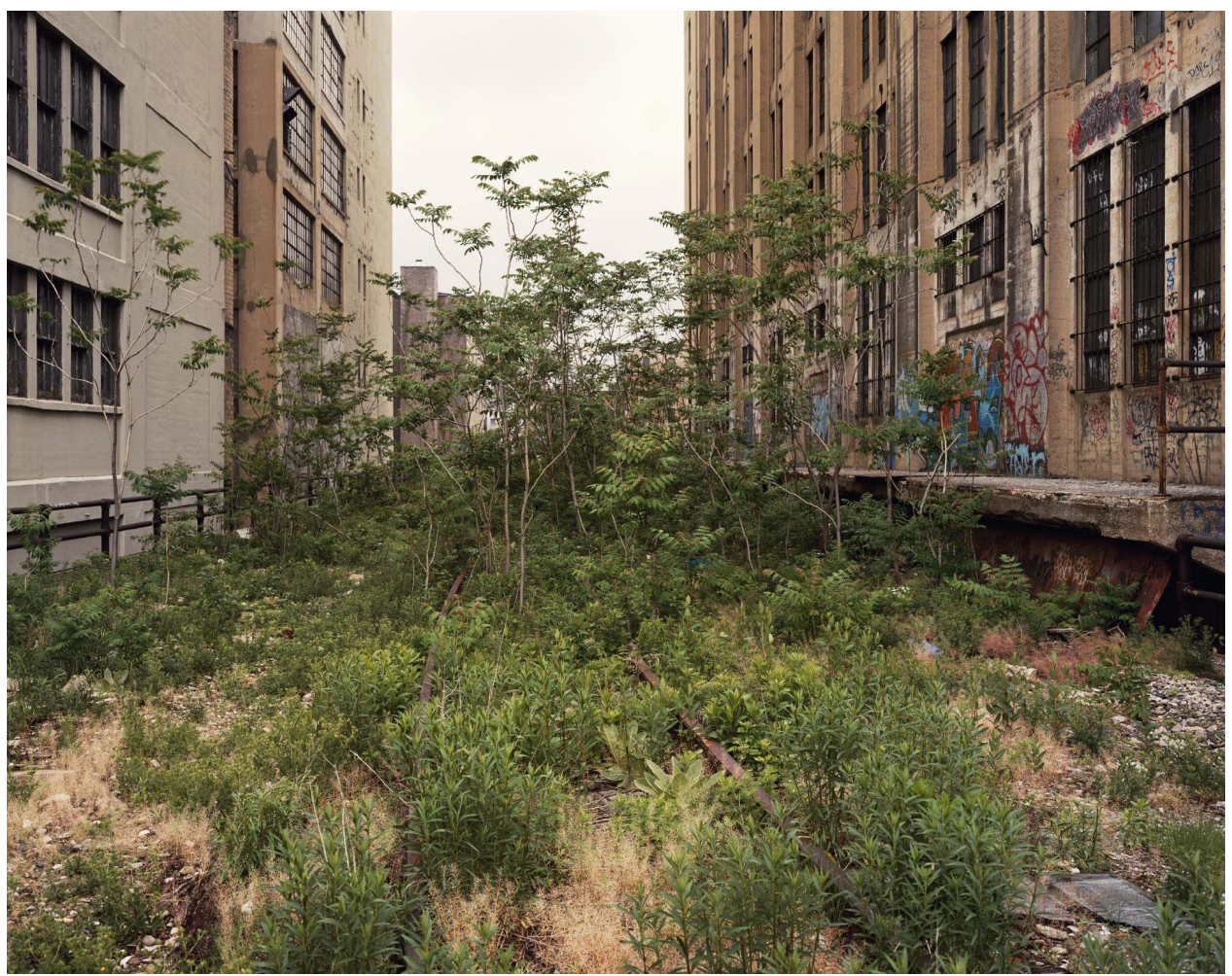

(c) Joel Sternfeld; Courtesy of the artist and Luhring Augustine, New York.

Le titre Walking the High Line insiste sur cette dimension itinérante. Le gérondif signe une action en cours, un processus dont la valeur est notifiée. La marche se présente donc comme une forme d'occupation du terrain, à valeur performative : en progressant sur la High Line, Sternfeld «fait» (au sens fort d'une réalisation) la promenade future. La marche du photographe est pèlerinage puisqu'il suit le trajet qui était celui des trains de marchandise, mais elle est aussi reconsidération du site, dès lors soumis à une appréciation de type esthétique. Francesco Careri utilise le néologisme "walkscapes " pour suggérer le pouvoir révélateur de la marche en matière de paysage (Careri F., 2013). En tant qu'expérience vécue de l'espace, la progression pédestre est, pour lui, tout à la fois « un acte perceptif et un acte créatif » (ibid. : 56). La marche sur la High Line propose également un point de vue inédit sur la ville : c'est souvent l'arrière des bâtiments qui est visible, un large panorama s'ouvrant sur la cité... Elle engendre bien un paysage spécifique.

La singularité de la promenade, ouverte aux visiteurs depuis 2009, tient à sa configuration surélevée fendant la ville, à la vue généreuse qu'elle ménage sur la cité, mais aussi aux choix qui ont été effectués dans sa conception même. Les rails ont été laissés et la végétation n'a pas été trop domestiquée; l'allure désordonnée qu'elle possède sur les photographies de Joel Sternfeld lui a été peu ou prou conservée, même si des bancs et des pistes en bois ont été disposés pour les promeneurs et que des expositions se trouvent organisées in situ. Le lieu est découvert à travers la temporalité du cheminement, mais le 
voyage dans l'espace est aussi voyage dans le temps, puisque l'aménagement du site a laissé la structure et les rails intacts, comme le désordre de la végétation qui témoigne du moment où la ligne était en friche, au côté des aménagements plus récents. L'espace, tel qu'il se présente aujourd'hui, est palimpseste des états successifs du site - dont il conserve des dépôts. Les lieux se présentent ainsi dotés d'une mémoire, qui constitue leur identité. Le passé se manifeste perpétuellement sous les pas des promeneurs; à cet égard, la High Line, telle qu'elle est aujourd'hui, rappelle peu ou prou les espaces paysagers du $\mathrm{XIX}^{\mathrm{e}}$ siècle où la présence de «fabriques » renvoyait à des temps révolus; mais celles-ci étaient artificielles, alors que les restes de la voie ferrée ont réellement connu d'autres usages, il y a peu.

Tandis que les architectes et les urbanistes de la période moderne concevaient des aménagements neufs qui faisaient fi des temps anciens pour délimiter ex nihilo un cadre nouveau pour les activités humaines, une plus grande attention est aujourd'hui accordée au contexte comme au lieu dans sa dimension historique et identitaire. La tendance est à la conservation, à la combinaison d'éléments hétérogènes renvoyant à différentes époques et à divers usages, à la préservation d'une stratification, à une attention portée aux symboles. À cet égard, les photographies de Joel Sternfeld ont joué un rôle car elles ont constitué une mémoire des aspects de la friche, au gré des saisons ; elles ont œuvré à son " artialisation » in visu, préfigurant une ultérieure "artialisation » in situ (Roger A., 1997). Elles ont, de la sorte, participé, à leur manière, à une des grandes entreprises de gentrification de la ville, puisque - comme l'a montré Michel Lussault - les antagonismes qui se manifestent aujourd'hui au sujet de l'occupation de certaines aires spatiales sont bien souvent l'expression de conflits de classes (Lussault M., 2009). Lors de la mobilisation pour la préservation du site, ce sont de fait des habitants aisés, des galeristes, des personnalités du monde de l'art et de la culture qui se mobilisent pour la conservation des vestiges de la voie ferrée.

\section{Conclusion}

L'attrait pour les ruines industrielles se présente, à la période contemporaine, comme un tropisme dont les raisons économiques et urbanistiques sont claires - la friche s'offrant comme un indice patent de mutations socio-économiques en cours, une invitation à l'incrémentation réussie des restes du passé avec des aménagements neufs, un éventuel défi en matière de mémoire. Des questions sociétales d'importance se trouvent immédiatement conviées par le traitement photographique de ce sujet. Dans le même temps, les ruines industrielles regorgent de motifs autour desquels peut s'incarner une forme de néo-pittoresque "déglingué ", ou encore un goût partagé pour le frisson de la catastrophe.

Mais d'autres approches photographiques sont possibles. Les travaux de Joel Sternfeld montrent en effet que le spectacle peut laisser place à la suggestion d'une expérience du lieu qui ouvre le champ de l'imagination et s'offre en partage au spectateur. Ce dernier peut ainsi s'approprier l'espace par procuration, l'habiter à sa manière, l'appréhender comme un paysage en devenir. C'est sans doute alors que s'ouvre la possibilité d'une requalification des lieux, d'un renouvellement des usages. Il faut cependant convenir que si l'aménagement actuel de la High Line ne tourne pas le dos au passé qui constitue l'identité même des sites, il en réserve peu ou prou la jouissance à certaines catégories sociales - ce qui constitue tout de même une étrange entorse à la mémoire. 


\section{BIBLIOGRAPHIE}

Adorno, Theodor et Horkheimer, Max, «La production industrielle des biens culturels », in La

Dialectique de la raison, Paris, Gallimard, 1974.

Benjamin, Walter, Paris, Capitale du XIX ${ }^{e}$ siècle. Le livre des Passages, 1927-1949, Paris, Éditions du Cerf, 1989.

Boeri, Stefano, L’Anti-ville, Paris, Manuella Éditions, 2013.

Caraion, Marta, Pour fixer la trace. Photographie, littérature et voyage au milieu du XIX siècle, Genève, Droz, 2003.

Careri, Francesco, Walkscapes. La marche comme pratique esthétique, Paris, Jacqueline Chambon, 2013.

Caron, François, Les deux révolutions industrielles du xxe siècle, Paris, Albin Michel, 1997.

Gilpin, William, Trois essais sur le beau pittoresque, sur les voyages pittoresques et sur l'art d'esquisser les paysages, suivi d'un poème sur la peinture de paysage, paru en 1792, traduit de l'anglais par le baron de Blumenstein [éd. orig. Three Essays On Picturesque Beauty] Paris, Éditions du Moniteur, coll. « Le Temps des jardins », 1982 [1799].

Jorion, Thomas, Îlots intemporels, Paris, Thomas Jorion, 2010.

Joshua, David et Robert Hammond, High Line. The Inside Story of New York City's Park in the Sky, New York, Farrar, Straus and Giroux, 2011.

Lussault, Michel, De la lutte des classes à la lutte des places, Paris, Grasset, 2009.

Lussault, Michel, L'Avènement du monde. Essai sur l'habitation humaine de la terre, Paris, Seuil, 2013.

Lyotard, Jean-François, La Condition postmoderne, Paris, Minuit, 1979.

Marchand, Yves et Meffre, Romain, Détroit, vestige du rêve américain, Göttingen, Steidl, 2010.

Méaux, Danièle, Voyages de photographes, Saint-Étienne, Publications de l'Université de SaintÉtienne, CIEREC - Travaux 141, 2009.

Merleau-Ponty, Maurice, Phénoménologie de la perception, Paris, Gallimard, 1985 [1945].

Paquot, Thierry, Un philosophe en ville, Paris, Infolio, 2011.

Roger, Alain, Court traité du paysage, Paris, Gallimard, 1997.

Rosa, Harmut, Accélération. Une critique sociale du temps, Paris, La Découverte, coll. « Théorie critique », 2010.

Smithson, Robert, « Entropy and the New Monuments » in Nancy Holt, The Writings of Robert Smithson, New York, New York University Press, 1979, p. 9-18.

Sternfeld, Joel, Walking the High Line, Göttingen, Steidl, 2001.

Vergara, Camilo José, American Ruins, The Monacelli Press, 2003. Voir le site de l'artiste : www.camilojosevergara.com, consulté le 10 janvier 2017.

Virilio, Paul, Ce qui arrive, Paris, Fondation Cartier, 2002. 


\section{RÉSUMÉS}

Le reflux de l'activité industrielle a entraîné la désaffection d'infrastructures importantes, souvent situées dans les centres urbains, et la présence de ces sites abandonnés pose problème aux citadins comme aux responsables territoriaux. S'ils signalent une crise économique aux effets sociaux graves, ils attirent les cinéastes ou les photographes qui aiment à mettre les ruines en images, esthétisant les formes incomplètes et les textures abîmées. Certains d'entre eux semblent même cultiver une certaine attraction pour le spectacle de la catastrophe. C'est le penchant de deux photographes français, Yves Marchand et Romain Meffre, dont les travaux consacrés à la ville de Détroit ont fait la notoriété. Bien loin de ces derniers, se situe la série photographique réalisée par Joel Sternfeld sur la High Line à New York, entre avril 2000 et juillet 2001. Le photographe américain, connu pour ses travaux paysagers en couleur, effectue ses prises de vue sur une voie ferroviaire en friche. Alors que le travail d'Yves Marchand et Romain Meffre suscite l'étonnement de l'observateur (presque devenu voyeur), les vues de Joel Sternfeld font partager au spectateur l'expérience de la déambulation. Dans ses images, la vitalité et la variété de la végétation, au gré du passage des saisons, s'imposent. Le livre publié en 2001 renvoie au geste d'appropriation que constitue la marche, préfigurant la destinée ultérieure du site. Le photographe milite, de fait, pour la conservation de la High Line et sa transformation en promenade.

The decline of industrial activity has led to many important infrastructures falling into disuse, structures often situated in urban centers, with the presence of these abandoned sites posing problems for both town-dwellers and local authorities. While they highlight an economic crisis with serious social repercussions, they also attract film-makers or photographers who like to display the ruins, aestheticising the fragmented forms and tatty textures. Certain of them even seem to cultivate a kind of attraction in the portrayal of catastrophes. Such are the inclinations of two French photographers, Yves Marchand and Romain Meffre, whose work devoted to the city of Detroit is renowned. Far from the work of the latter we find the photographic series produced by Joel Sternfeld on the High Line in New York, between April 2000 and July 2001. The American photographer, known for his work in colour on landscapes, took a series of shots of an abandoned elevated railway line. While the work of Yves Marchand and Romain Meffre provokes a certain astonishment on the part of the observer (who becomes almost a voyeur), the shots of Joel Sternfeld allow the spectator to share the experience of his strolls along the line. What emerges in these images is the vitality and diversity of the vegetation, changing with the seasons. The book, published in 2001, relates to the gesture of appropriation which the walk inspires, presaging the future destiny of the site. The photographer is actually campaigning for the preservation of the High Line and its conversion to a walkway.

La disminución de la actividad industrial acarreó el desmoronamiento de infraestructuras importantes, a menudo situadas en los centros urbanos. Esas instalaciones abandonadas plantean numerosos problemas a los ciudadanos como a los responsables de la gestión territorial. Si indican una crisis económica de efectos sociales graves, atraen a los cineastas y los fotógrafos a quienes les gusta sacar fotos de las ruinas, dándole un tratamiento estético a las formas incompletas y las texturas estropeadas. Hasta parecen cultivar algunos de ellos cierta atracción por el espectáculo de la catástrofe. Es la tendencia de dos fotógrafos franceses, Yves Marchand y Romain Meffre, cuya notoriedad se debe a sus obras dedicadas a la ciudad de Detroit. Dista mucho de ellas la serie fotográfica realizada por Joel Sternfeld sobre la High Line de Nueva York, entre abril de 2000 y julio de 2001. El fotógrafo norteamericano, conocido por sus paisajes en color, toma sus fotos en una vía ferroviaria en desuso. Cuando el trabajo de Marchand y Meffre suscita 
la sorpresa del espectador (que limita con el voyeur), la obra de Sternfeld le permite al espectador compartir la experiencia de la deambulación a lo largo de las cuatro estaciones del año. Lo que se impone es la vitalidad y la variedad de la vegetación. El libro remite al movimiento de apropiación que constituye el andar, prefigurando el destino ulterior de las instalaciones. En realidad, Sternfeld aboga por la conservación de la High Line y su transformación en paseo.

\section{INDEX}

Mots-clés : friche, paysage, marche, photographie, ruine, spectacle

Keywords : wasteland, landscape, walk, photography, ruin, show

Palabras claves : terrenos baldíos industriales, paisaje, andar, fotografía, ruina, espectáculo

\section{AUTEUR}

\section{DANIÈLE MÉAUX}

Professeur des Universités en esthétique et sciences de l'art à Saint-Étienne, Danièle Méaux est spécialiste de la photographie contemporaine. Elle est l'auteur de La Photographie et le temps. Le déroulement temporel dans l'image photographique (PUP, 1997), Voyages de photographes (PUSE, 2009), Géo-photographies. Une approche renouvelée des territoires (Filigranes, 2015). Elle prépare actuellement un ouvrage sur les démarches de l'enquête, telles qu'elles sont aujourd'hui mobilisées par les photographes. Elle est rédacteur en chef de la revue en ligne Focales. daniele.meaux@univ-st-etienne.fr 